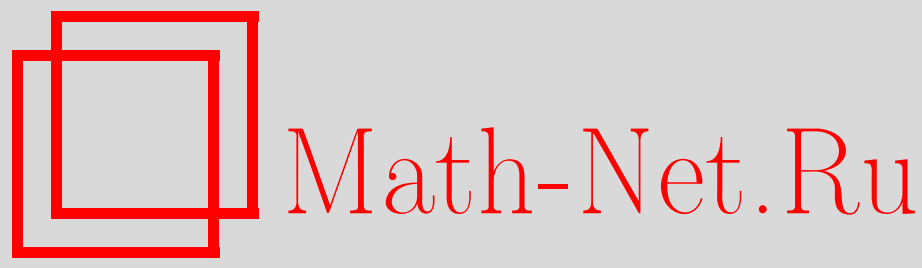

В. Г. Марихин, Действие как инвариант преобразований Беклунда лагранжевых систем, ТМФ, 2015, том 184, номер 1, 71-78

DOI: https://doi.org/10.4213/tmf8890

Использование Общероссийского математического портала Math-Net.Ru подразумевает, что вы прочитали и согласны с пользовательским соглашением http://www.mathnet.ru/rus/agreement

Параметры загрузки:

IP : 54.224 .187 .69

26 апреля 2023 г., 13:20:59

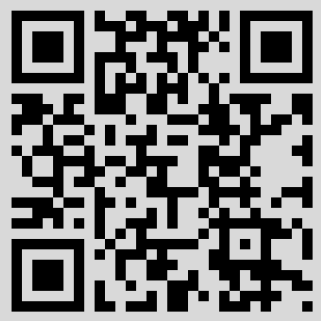




\title{
ДЕЙСТВИЕ КАК ИНВАРИАНТ ПРЕОБРАЗОВАНИЙ БЕКЛУНДА ЛАГРАНЖЕВЫХ СИСТЕМ
}

\begin{abstract}
Построена общая теория преобразований Беклунда лагранжевых систем при условии, что действие сохраняется при применении этих преобразований. Обсуждаются известные преобразования Беклунда для классических солитонных уравнений с точки зрения этого подхода. Получено новое преобразование Беклунда для уравнения Цицейки.
\end{abstract}

Ключевые слова: интегрируемые системы, канонические преобразования.

DOI: $10.4213 / \operatorname{tmf} 8890$

\section{1. ВВЕДЕНИЕ}

Преобразования Беклунда (ПБ), т. е. преобразования, позволяющие получать новые решения данной динамической системы, являются одним из эффективных методов анализа системы на интегрируемость. Рассматривая композицию ПБ, можно построить дифференциально-разностные системы - интегрируемые цепочки, алгебраически эквивалентные рассматриваемым динамическим системам. Если данная динамическая система является гамильтоновой, то ПБ суть канонические преобразования, сохраняющие гамильтониан, а значит, и действие (в силу каноничности преобразования).

Мы будем рассматривать $(1+1)$-мерные лагранжевые системы вида

$$
S=\int d t L, \quad L=\int d x l\left(q_{t}, q, q_{x}, q_{x x}, \ldots\right) .
$$

Следует отдельно рассматривать случаи линейной и нелинейной зависимости от $q_{t}$ плотности лагранжиана $l$. В нелинейном случае мы будем рассматривать в основном квадратичные по $q_{t}$ плотности лагранжиана $l$. Данный подход был ранее развит в работе [1], а также в работах [2] и [3].

В настоящей работе проведена классификация возможных типов ПБ в линейном случае (когда коэффициенты при $q_{t}$ в плотности лагранжиана $l$ зависят только от $q_{x}$ ). Все известные системы в линейном случае обладают этим свойством.

${ }^{*}$ Институт теоретической физики им. Л. Д. Ландау РАН, Москва, Россия. E-mail: mvg@itp.ac.ru 
Показано, что лагранжиан соответствующей цепочки ПБ выражается через производящую функцию канонического преобразования данного ПБ. Найдено новое ПБ для уравнения Цицейки, которое не зависит от $q_{t}$ в отличие от ранее известных ПБ.

Мы будем пользоваться обозначением $\hat{A}$ для величины $A$ после применения ПБ.

\section{2. ГАМИЛЬТОНОВА ТЕОРИЯ ПРЕОБРАЗОВАНИЙ БЕКЛУНДА}

2.1. Канонические преобразования. Рассмотрим случай нелинейной зависимости плотности лагранжиана от $q_{t}$. Большая часть примеров, которые мы будем рассматривать, связана с уравнениями из известного списка (см. работу [4]) систем вида

$$
\mathbf{u}_{t}=A(\mathbf{u}) \mathbf{u}_{x x}+B\left(\mathbf{u}, \mathbf{u}_{x}\right), \quad \operatorname{det} A \neq 0, \quad \mathbf{u}=\left(u^{1}, u^{2}\right),
$$

обладающих высшими законами сохранения. В частности, этот класс содержит нелинейное уравнение Шредингера и уравнение Буссинеска

$$
q_{t t}+q_{x x x x}+q_{x} q_{x x}=0 .
$$

Эта теория является достаточно общей и охватывает широкий класс лагранжевых систем вида (1). В частности, лагранжианы с плотностями

$$
l=\left(q_{t}+q_{x x}\right)^{2}+q_{x}^{3}, \quad l=\frac{1}{2}\left(q_{t}+q_{x x}-q_{x}^{2}\right)^{2}, \quad l=\frac{\left(q_{t}+q_{x x}+r^{\prime}(q) / 2\right)^{2}}{4\left(q_{x}^{2}+r(q)\right)}+\frac{1}{12} r^{\prime \prime}(q)
$$

отвечают соответственно уравнению Буссинеска, нелинейному уравнению Шредингера и анизотропной модели Ландау-Лифшица. Плотность $h[p, q]=h\left(p, q_{x x}, q_{x}, q\right)$ гамильтониана $H=\int d x h$ связана с плотностью лагранжиана $l$ стандартными формулами

$$
h=q_{t} l_{q_{t}}-l, \quad l=p h_{p}-h, \quad\left(p=l_{q_{t}}\right) .
$$

ПБ, переводящее решение гамильтоновой динамической системы

$$
q_{t}=\frac{\delta H[p, q]}{\delta p}, \quad p_{t}=-\frac{\delta H[p, q]}{\delta q}
$$

снова в решение, определяется как каноническое преобразование $(p, q) \rightarrow(\hat{p}, \hat{q})$ с производящей функцией $F[q, \hat{q}]$ :

$$
p=\frac{\delta F[q, \hat{q}]}{\delta q}, \quad \hat{p}=-\frac{\delta F[q, \hat{q}]}{\delta \hat{q}} .
$$

ОПРЕДЕЛЕНИЕ 1. Функция $F[q, \hat{q}]=\int d x f\left(q, q_{x}, \ldots, \hat{q}, \hat{q}_{x}, \ldots\right)$ называется производящей функцией канонического ПБ для динамической системы (2), если существует функция $\sigma[q, \hat{q}]$ такая, что

$$
h[\hat{p}, \hat{q}]-h[p, q]=\frac{d}{d x} \sigma[q, \hat{q}],
$$

где $p, \hat{p}$ определены формулами (3).

В силу этого определения и формул

$$
p_{n}=\frac{\delta F\left[q_{n}, q_{n+1}\right]}{\delta q_{n}}=-\frac{\delta F\left[q_{n-1}, q_{n}\right]}{\delta q_{n}} \quad \Longrightarrow \quad \frac{\delta F\left[q_{n}, q_{n+1}\right]}{\delta q_{n}}+\frac{\delta F\left[q_{n-1}, q_{n}\right]}{\delta q_{n}}=0
$$


производящая функция определяет лагранжиан цепочки ПБ:

$$
\mathcal{L}=\sum_{n} F\left[q_{n}, q_{n+1}\right]=0
$$

(ср. работу [5], аналогичные цепочки рассмативались в статье [6], см. также [7]).

Покажем, что действие сохраняется при применении ПБ. Действительно,

$$
\int d t \int d x\left(\hat{p} \hat{q}_{t}-p q_{t}\right)=-\int d t \frac{d}{d t} F[q, \hat{q}]=0 .
$$

В силу соотношения (4) $\widehat{H}-H=0$, следовательно, $\widehat{S}-S=0$. Подставляя выражения (3) для импульсов в (4), мы получим определяющее уравнение для производящей функции $F$ и гамильтониана $H$.

Поскольку классификация рассматриваемых систем была проведена в работе [1], здесь мы приведем только наиболее интересные примеры (см. также статью [8]) в случае $f_{q_{x}, \hat{q}_{x}}=0$.

ПримеР 1. Система с плотностью гамильтониана

$$
h=-p q_{x x}+p q_{x}^{2}+p^{2}
$$

имеет следующую плотность канонического ПБ:

$$
f=-\frac{1}{2} \hat{q}_{x}^{2}+\alpha q_{x} e^{\hat{q}-q}+\frac{1}{2} \alpha^{2} e^{2(\hat{q}-q)}+\beta e^{\hat{q}-q} .
$$

При $\alpha=0, \beta=1$ получаем лагранжиан цепочки Тоды (5), а в общем случае лагранжиан магнитной цепочки Тоды [8]. Отметим, что система (6) изоморфна нелинейному уравнению Шредингера: при замене $q=-\ln \psi, p=\psi^{*} \psi$ плотность гамильтониана принимает вид

$$
h=-\psi_{x}^{*} \psi_{x}+\left(\psi^{*} \psi\right)^{2} .
$$

В данном случае скобки Пуассона канонические: $\left\{\psi^{*}(x), \psi\left(x^{\prime}\right)\right\}=\delta\left(x-x^{\prime}\right)$.

ПримеР 2. Система Леви с плотностью гамильтониана

$$
h=-p q_{x x}+p q_{x}^{2}+p^{2} q_{x}
$$

имеет следующую плотность канонического ПБ:

$$
f=\hat{q}_{x} \ln \hat{q}_{x}-q_{x} \ln \left(\alpha e^{\hat{q}-q}+\beta\right)+\delta e^{\hat{q}-q} .
$$

При $\alpha=0, \beta=1, \delta=1$ мы получаем лагранжиан цепочки Вольтерра (5), а в общем случае - лагранжиан магнитной цепочки Вольтерра [8].

ПримеР 3. Модель Ландау-Лифшица

$$
h=-p q_{x x}-p^{2} q_{x}^{2}-p^{2} r(q)-\frac{1}{2} p r^{\prime}(q)-\frac{1}{12} r^{\prime \prime}(q)
$$

имеет следующие плотности канонического ПБ:

$$
f=\frac{1}{\sqrt{r(q)}} \operatorname{arctg} \frac{q_{x}}{\sqrt{r(q)}}-\ln \left(q_{x}^{2}+r(q)\right)+\ln (\hat{q}-q)
$$


для немагнитной цепочки ПБ модели Ландау-Лифшица и

$$
f=\frac{1}{\sqrt{r(q)}} \operatorname{arctg} \frac{q_{x}}{\sqrt{r(q)}}-\ln \left(q_{x}^{2}+r(q)\right)+\frac{\hat{q}_{x}}{\sqrt{r(\hat{q})}} \operatorname{arctg} \frac{\mathrm{h}_{q}}{\sqrt{r(\hat{q})}}+\frac{1}{2} \ln \mathrm{h}
$$

для магнитной цепочки, где в обоих случаях $r(u)$ есть многочлен не выше четвертой степени, $\mathrm{h}(u, v)$ - симметрический полином не выше второй степени по каждой переменной, причем $r(u)$ является его дискриминантом,

$$
r(u)=-\mathrm{h}_{v}^{2}+2 \mathrm{hh}_{v v}, \quad \mathrm{~h}(u, v)=\mathrm{h}(v, u), \quad \mathrm{h}_{u u u}=0 .
$$

Гамильтонова форма соответствующих цепочек (см. работу [9])

$$
u_{n, x}=\frac{2 \mathrm{~h}\left(u_{n}, v_{n}\right)}{u_{n+1}-v_{n}}+\mathrm{h}_{n, v_{n}}, \quad v_{n, x}=\frac{2 \mathrm{~h}\left(u_{n}, v_{n}\right)}{u_{n}-v_{n-1}}-\mathrm{h}_{n, u_{n}}
$$

непосредственно связана [10] с известной $X Y Z$-цепочкой Склянина [11].

2.2. Уравнения типа $K_{д} \Phi$, sh-Гордон и др. Пусть

$$
\mathcal{L}=\int d x\left(q_{t} g\left(q_{x}\right)-H\left(q, q_{x}, \ldots\right)\right) .
$$

Мы рассматриваем случай, когда функция $g$ зависит только от $q_{x}$, поскольку зависимость от старших производных привела бы к сильно нелокальным уравнениям Эйлера-Дарбу. Что касается явной зависимости $g$ от $q$, то в ряде случаев эту зависимость можно устранить точечным преобразованием, например

$$
q_{t} q_{x}^{\alpha} g(q)=Q_{t} Q_{x}^{\alpha}, \quad d Q=(g(q))^{1 /(\alpha+1)} d q .
$$

Сдвиг $g \rightarrow g+s(q)$ не влияет на уравнения движения, поскольку величина $s(q) q_{t}$ является полной производной. В более сложных случаях допустимые $g$ с явной зависимостью от $q$ неизвестны.

Условие $\hat{L}-L \in \operatorname{Im} D_{t}+\operatorname{Im} D_{x}$ разбивается на два:

$$
\hat{q}_{t} g\left(\hat{q_{x}}\right)-q_{t} g\left(q_{x}\right) \in \operatorname{Im} D_{t}+\operatorname{Im} D_{x}, \quad \widehat{H}-H \in \operatorname{Im} D_{x} .
$$

Перепишем первое из условий (8) в явном виде

$$
\hat{q}_{t} g\left(\hat{q_{x}}\right)-q_{t} g\left(q_{x}\right)=\partial_{t}\left(q_{x} v(q, \hat{q})+u(q, \hat{q})\right)-\partial_{x}\left(q_{t} v(q, \hat{q})\right) .
$$

Приравнивая коэффициенты при $q_{t}$ и $\hat{q}_{t}$, получаем систему уравнений

$$
g\left(\hat{q_{x}}\right)=q_{x} v_{\hat{q}}+u_{\hat{q}}, \quad g\left(q_{x}\right)=\hat{q}_{x} v_{\hat{q}}-u_{q} .
$$

Положим

$$
s(w)=\frac{g(w)+u_{q}}{v_{\hat{q}}}, \quad \tilde{s}(w)=\frac{g(w)-u_{\hat{q}}}{v_{\hat{q}}} .
$$

Функции $s(w), \tilde{s}(w)$ зависят также от $q, \hat{q}$ и, возможно, от спектрального параметра, но для краткости мы пишем, что $g(w)$ зависит только от $w$.

ПБ можно записать в виде

$$
q \rightarrow \hat{q} \rightarrow q, \quad \hat{q}_{x}=s\left(q_{x}\right), \quad q_{x}=\tilde{s}\left(\hat{q}_{x}\right) \quad \Longrightarrow \quad s(\tilde{s}(w))=w, \quad \tilde{s}(s(w))=w .
$$


Очевидно, что $\partial_{\lambda}(s(\tilde{s}(w)))=0, \partial_{\lambda}(\tilde{s}(s(w)))=0$, где $\lambda-$ спектральный параметр (либо $q$, либо $\hat{q}$ ). Имеем

$$
\partial_{\lambda}(s(\tilde{s}(w)))=\left(\frac{1}{v_{\hat{q}}}\right)_{\lambda} g(\tilde{s}(w))+\left(\frac{u_{q}}{v_{\hat{q}}}\right)_{\lambda}+s^{\prime}(\tilde{s}(w))\left[\left(\frac{1}{v_{\hat{q}}}\right)_{\lambda} g(w)-\left(\frac{u_{\hat{q}}}{v_{\hat{q}}}\right)_{\lambda}\right] .
$$

Полагая $\xi=\tilde{s}(w)$ и используя $(10)$, получаем $s^{\prime}(\xi)=g^{\prime}(\xi) / v_{\hat{q}}$. Подставим $g(w)$, выразив ее из второго уравнения (10), в результате имеем

$$
g^{\prime}(\xi)\left(\epsilon_{1} \xi+\epsilon_{2}\right)+\left(\epsilon_{1} g(\xi)+\epsilon_{3}\right)=0 \quad \Longrightarrow \quad g(\xi)=\frac{-\epsilon_{3} \xi+\epsilon_{4}}{\epsilon_{1} \xi+\epsilon_{2}}
$$

где

$$
\epsilon_{1}=\frac{1}{v_{\hat{q}}}, \quad \epsilon_{2}=-\frac{u_{\hat{q}, \lambda}}{v_{\hat{q}}^{2}}, \quad \epsilon_{3}=\left(\frac{u_{q}}{v_{\hat{q}}}\right)_{\lambda} .
$$

В случае $\epsilon_{1}=0$ функция $g(\xi)$ линейна по $\xi$. Применяя преобразования сдвига и растяжения (которые не влияют на уравнения движения), можно выбрать $g(\xi)=\xi$. В случае $\epsilon_{1} \neq 0$, используя преобразования сдвига и растяжения, можно получить $g(\xi)=1 /\left(\xi-\xi_{0}\right)$. На данный момент не существует примеров, соответствующих случаю $\xi_{0} \neq 0$. Таким образом, остаются два случая: либо $g(\xi)=\xi$, либо $g(\xi)=1 / \xi$.

Случаю $g(\xi)=\xi$ соответствует требование

$$
\iint d t d x\left(\hat{q}_{x} \hat{q}_{t}-q_{x} q_{t}\right)=\iint d t d x\left(\hat{q}_{x}+q_{x}\right)\left(\hat{q}_{t}-q_{t}\right),
$$

следовательно, ПБ имеет вид

$$
\hat{q}_{x}+q_{x}=\frac{\delta F}{\delta(\hat{q}-q)},
$$

где $F=\int d x f\left(\hat{q}-q, \hat{q}_{x}-q_{x}, \hat{q}_{x x}-q_{x x}, \ldots\right)$. В простейших случаях $f$ зависит только от $\hat{q}-q$.

ПримеР 4. Рассмотрим уравнение КдФ

$$
q_{t}=q_{x x x}-6 q_{x}^{2} \quad \Longrightarrow \quad D_{x}\left(q_{t}\right)=\frac{\delta H}{\delta q}, \quad H=\int d x h, \quad h=\frac{1}{2} q_{x x}^{2}+2 q_{x}^{3} .
$$

Учитывая, что $\hat{h}-h$ должна быть полной производной в силу $(11)$, получаем

$$
f(\hat{q}-q)=\frac{1}{3}(\hat{q}-q)^{3}+\beta(\hat{q}-q) .
$$

Подставляя данное равенство в (11), получаем окончательно ПБ в виде

$$
\hat{q}_{x}+q_{x}=(\hat{q}-q)^{2}+\beta .
$$

Параметр $\beta$ можно выбирать разным на каждом шаге. Введя обозначение $f=\hat{q}-q$, получим одевающую цепочку [12].

Данный пример допускает обобщение на $(2+1)$-мерный случай.

ПримеР 5. Лагранжиан и уравнение Кадомцева-Петвиашвили имеют вид

$$
\begin{gathered}
L=\int d x\left[q_{x} q_{t}-q_{x x}^{2}-2 q_{x}^{3}-3 \alpha^{2} q_{y}^{2}\right], \\
q_{x}=u, \quad \partial_{x}\left(u_{t}+u_{x x x}-6 u u_{x}\right)=3 \alpha^{2} u_{y y} .
\end{gathered}
$$


ПБ можно получить аналогично уравнению КдФ, причем спектральный параметр заменяется на нелокальный член:

$$
q_{x}+\hat{q}_{x}=\frac{1}{2}(\hat{q}-q)^{2}+Z, \quad Z=\gamma \partial_{x}^{-1} \partial_{y}(\hat{q}-q), \quad \gamma=i \alpha .
$$

Лагранжиан цепочки, соответствующей уравнению (12), имеет вид

$$
l=\sum_{n}\left[q_{n+1, x} q_{n}+\frac{1}{6}\left(q_{n+1}-q_{n}\right)^{3}+\frac{\gamma}{2}\left(q_{n+1}-q_{n}\right) \partial_{x}^{-1} \partial_{y}\left(q_{n+1}-q_{n}\right)\right] .
$$

ПримеР 6. Рассмотрим уравнение sh-Гордон

$$
q_{x t}=\operatorname{sh} q, \quad h=-2 \operatorname{ch} q .
$$

Выражение

$$
\operatorname{ch} \hat{q}-\operatorname{ch} q=2 \operatorname{sh} \frac{\hat{q}+q}{2} \operatorname{sh} \frac{\hat{q}-q}{2}
$$

является полной производной, если ПБ уравнения sh-Гордон имеет вид

$$
\hat{q}_{x}+q_{x}=\beta \operatorname{sh} \frac{\hat{q}-q}{2} .
$$

В этом уравнении $x$ и $t$ эквивалентны, отсюда получаем $t$-ПБ

$$
\hat{q}_{t}-q_{t}=\frac{4}{\beta} \operatorname{sh} \frac{\hat{q}+q}{2} .
$$

Рассмотрим теперь случай $g(\xi)=1 / \xi$. Анализ уравнений (9) приводит к следующей форме ПБ:

$$
\hat{q}_{x} q_{x}=\frac{1}{v_{\hat{q}}} .
$$

Этой форме ПБ соответствует следующий пример.

ПримеР 7. Лагранжиан для уравнения Кричевера-Новикова

$$
q_{t}=q_{x x x}-\frac{3}{2 q_{x}}\left(q_{x x}^{2}-r(q)\right)+c q_{x}
$$

записывается как

$$
L=\int d x\left[\frac{q_{t}}{q_{x}}+\frac{r(q)-q_{x x}^{2}}{q_{x}^{2}}\right], \quad r^{(V)}(q)=0 .
$$

Вид ПБ в этом случае (см. выше) таков:

$$
q_{x} \hat{q}_{x}=\mathrm{h}(q, \hat{q})=\left(v_{\hat{q}}(q, \hat{q})\right)^{-1} .
$$

Условие сохранения гамильтониана приводит к тому, что $\mathrm{h}(q, \hat{q})$ - полином второй степени по $q$ и $\hat{q}$ такой, что $r(q)=2 \mathrm{hh}_{\hat{q} \hat{q}}-\mathrm{h}_{\hat{q}}^{2}$. ПБ уравнения Кричивера-Новикова было получено в работе [13]. Лагранжиан цепочки ПБ имеет вид

$$
L=\sum_{n} v\left(q_{n-1}, q_{n}\right) q_{n, x}, \quad v(q, \hat{q})=\frac{2}{\sqrt{r(q)}} \operatorname{arctg} \frac{\mathrm{h}_{\hat{q}}(q, \hat{q})}{\sqrt{r(q)}} .
$$




\section{3. ПРЕОБРАЗОВАНИЯ БЕКЛУНДА УРАВНЕНИЯ ЦИЦЕЙКИ}

Уравнение Цицейки [14] было получено также в статье [15] как интегрируемый случай гиперболических уравнений типа sh-Гордон. Уравнение Цицейки является лагранжевым:

$\phi_{x, t}=e^{-2 \phi}-e^{\phi} \quad \Longleftrightarrow \quad \delta S=0, \quad S=\int d x d t\left[\phi_{x} \phi_{t}-h(\phi)\right], \quad h(\phi)=2 e^{\phi}+e^{-2 \phi}$.

ПБ есть каноническое преобразование (11) $b=\delta F[A] / \delta A$, где

$$
A=\hat{\phi}-\phi, \quad B=\hat{\phi}+\phi, \quad a=\hat{\phi}_{x}-\phi_{x}, \quad b=\hat{\phi}_{x}+\phi_{x} .
$$

Мы ищем ПБ в наиболее простой форме, возможной в данном случае:

$$
F=\int d x f(a, A) \quad \Longleftrightarrow \quad b=f_{A}-a_{x} f_{a a}-a f_{a A} .
$$

Каноническое преобразование (13) является ПБ в том и только в том случае, когда

$$
h(\hat{\phi})-h(\phi)=2 e^{B / 2} g^{\prime}(A)-e^{-B} g^{\prime}(A) g(A)=\partial_{x} W(a, A, B),
$$

где

$$
g(A)=e^{A / 2}+e^{-A / 2} .
$$

Можно удовлетворить уравнение (14) и найти производящую функцию $F(a, A)$, если выполнены условия

$$
\partial_{x}\left[e^{B / 2} Z(a, A)\right]=-e^{B / 2} g^{\prime}(A), \quad \partial_{x}\left[e^{-B} R(a, A)\right]=-e^{-B} g^{\prime}(A) g(A) .
$$

Первое условие дает

следовательно,

$$
f_{a}=2 \ln Z, \quad f_{A}=-2 \frac{g^{\prime}(A)}{Z},
$$

$$
g^{\prime}(A) Z_{a}-Z_{A} Z=0 \quad \Longleftrightarrow \quad g(A)+a Z=s(Z) .
$$

Второе условие определяет неизвестную функцию $s(Z)$ :

$$
s(Z)=\nu Z^{3}, \quad R=-\frac{g^{2}(A)}{\nu Z^{2}} .
$$

Итак, находим $Z(a, A)$ из кубического уравнения $\nu Z^{3}=g(A)+a Z$, интегрируем уравнение $f_{a}=2 \ln Z$ и находим плотность производящей функции ПБ $f(a, A)$. Другими словами,

$$
-\frac{b}{2} Z=Z_{x}+g^{\prime}(A), \quad Z_{x}=a Z_{A}+a_{x} Z_{a}
$$

где $Z(a, A)$ такова, что $\nu Z^{3}=a Z+g(A)$.

ПБ уравнения Цицейки в довольно громоздкой форме было получено в работе [16]. По-видимому, это преобразование не приводится к ПБ, полученным выше. Выведенная в настоящей статье форма является новым примером ПБ в виде (11). Кроме того, автору недавно стало известно [17], что получена еще одна форма ПБ для уравнения Цицейки. Автор благодарен А. В. Михайлову и В. В. Соколову за то, 
что они показали, что полученное выше ПБ для уравнения Цицейки может быть приведено в точности к преобразованию, полученному ими независимо.

В заключение отметим, что на основе полученных ПБ можно развить теорию преобразований, например, на основе одевающей цепочки [12]

$$
v_{i, x}+v_{i+1, x}=\left(v_{i}-v_{i+1}\right)^{2}-\beta_{i}, \quad f_{j}=v_{j}-v_{j+1} .
$$

Такой подход был развит, например, в работах [6], [8], [10]. Было бы интересно применить построенную теорию к случаю функции $g\left(q_{x}, q\right)$ из $(7)$.

Благодарности. Автор признателен участникам семинара "Математическая физика" ИТФ им. Л. Д. Ландау РАН за полезные обсуждения. Работа выполнена при частичной поддержке РФФИ (грант № 13-01-00402).

\section{Список литературы}

[1] В.Э. Адлер, В. Г. Марихин, А. Б. Шабат, ТМФ, 129:2 (2001), 163-183.

[2] V.G. Marikhin, A. B. Shabat, "Hamiltonian theory of Bäcklund transformations", Optical Solitons: Theoretical Challenges and Industrial Perspectives (Les Houches Workshop, September 28 -October 2, 1998), Centre de Physique des Houches, 12, eds. V. E. Zakharov, S. Wabnitz, Springer, Berlin, 1999, 19-29.

[3] В. Г. Марихин, Писъма в ЖЭТФ, 66:11 (1997), 673-68.

[4] А. В. Михайлов, А. Б. Шабат, Р. И. Ямилов, УМН, 42:4(256) (1987), 3-53.

[5] В. Э. Адлер, А. Б. Шабат, ТМФ, 112:2 (1997), 179-194.

[6] A. N. Leznov, A. B. Shabat, R. I. Yamilov, Phys. Lett. A, 174:5-6 (1993), 397-402.

[7] R. I. Yamilov, "Classification of Toda type scalar lattices", Nonlinear Evolution Equations and Dynamical Systems (NEEDS'92) (Dubna, Russia, 6-17 July, 1992), eds. V. G. Makhankov, O. Pashaev, I. Puzynin, World Sci., Singapore, 1993, 423-431.

[8] В. Г. Марихин, А. Б. Шабат, ТМФ, 118:2 (1999), 217-228.

[9] А. Б. Шабат, Р. И. Ямилов, Алгебра и анализ, 2:2 (1990), 183-208.

[10] В. Э. Адлер, ТМФ, 124:1 (2000), 48-61.

[11] Е. К. Склянин, Функи. анализ и его прил., 16:4 (1982), 27-34.

[12] А. П. Веселов, А.Б. Шабат, Функи. анализ и его прил., 27:2 (1993), 1-21.

[13] V. E. Adler, Internat. Math. Res. Notes, 1998, № 1, 1-4.

[14] M. G. Tzitzéica, Rendiconti del Circolo Matematico di Palermo, 25:1 (1907), 180-187.

[15] А. В. Жибер, А. Б. Шабат, Докл. АН СССР, 247:5 (1979), 1103-1107.

[16] С. С. Сафин, Р. А. Шарипов, ТМФ, 95:1 (1993), 146-159.

[17] А. В. Михайлов, В.В.Соколов, устное сообщение, 2015. 